\title{
Gravitational wave amplification of seed magnetic fields
}

\author{
Christos G. Tsagas ${ }^{1}$, Peter K. S. Dunsby ${ }^{1,2}$ and Mattias Marklund ${ }^{3}$ \\ ${ }^{1}$ Department of Mathematics and Applied Mathematics, University of Cape Town, Rondebosch 7701, South Africa \\ ${ }^{2}$ South African Astronomical Observatory, Observatory 7925, Cape Town, South Africa \\ ${ }^{3}$ Department of Electromagnetics, Chalmers University of Technology, SE-412 96 Göteborg, Sweden
}

\begin{abstract}
We discuss how gravitational waves could amplify seed magnetic fields to strengths capable of supporting the galactic dynamo. We consider the interaction of a weak magnetic field with gravity wave distortions in almost FRW cosmologies and find that the magnitude of the original field is amplified proportionally to the wave induced shear anisotropy and, crucially, proportionally to the square of the field's initial scale. The latter makes our mechanism particularly efficient when operating on superhorizon sized magnetic fields, like those produced during inflation. In that case, the achieved amplification can easily boost magnetic strengths, which may still lie relatively close to the galactic dynamo lower limits, well within the currently accepted range.
\end{abstract}

PACS number(s): 98.80.Hw, 04.30.-w, 98.80.Cq

\section{Introduction}

Large scale magnetic fields, with strengths between $10^{-7}$ and $10^{-5} G$, have been repeatedly observed in spiral and disc galaxies, in galaxy clusters as well as in high redshift condensations [1]. Despite their established widespread presence, however, the origin of cosmic magnetic fields remains a mystery and is still a matter of debate [2]. Over the years, a number of possible solutions has been proposed, ranging from eddies and density fluctuations in the early plasma to cosmological phase-transitions, inflationary and superstring inspired scenarios (see [3, 4] for a representative list). Historically, studies of magnetogenesis were motivated by the need to explain the origin of the large-scale galactic fields. Typical spiral galaxies have magnetic fields of the order of a few $\mu \mathrm{G}$ coherent over the plane of their disc. The structure of these fields, particularly those in spiral galaxies, strongly suggests that they have been generated and sustained by a dynamo mechanism [5]. Although the efficiency of the mechanism has been critisised, it is generally believed that galactic dynamos can substantially amplify preexisting weak magnetic fields by combining the turbulent motion of the ionised gas with the differential rotation of the galaxy. The origin of the required seed fields, however, is still elusive. They could be the result of local astrophysical processes, such as buttery and vorticity effects, or the remnants of a large scale primordial magnetic field. Provided that the nonlinear dynamo amplification is efficient, the seeds can be as low as $\sim 10^{-23} G$ at present [6]. For a spatially flat universe dominated by "dark-energy", namely by a cosmological constant or quintessence, the aforementioned lower limit is further relaxed down to $\sim 10^{-30} G$ [7]. In the absence of a dynamo mechanism, however, magnetic seeds of the order of $10^{-12} G$, or even $10^{-8} G$, are required. The coherence scale of the seed field is an additional issue. Typically, galactic dynamos require a minimum 
coherence length comparable to the dimensions of the largest turbulent eddy, which is of the order of $100 \mathrm{pc}$, to guarantee the stability of the amplification process [8].

The attractiveness of primordial magnetic fields lies in the fact that they can readily explain both the fields seen in nearby galaxies as well as those detected in galaxy clusters and highly redshifted condensations. There have been numerous attempts to generate early, pre-recombination, magnetic fields by exploiting the different out-of-equilibrium epochs that are believed to have taken place between the end of the inflationary era and decoupling. In all these scenarios, however, the causal nature of the generating mechanisms means that the coherence scale of the induced seed fields is unacceptably small. A process known as "inverse cascading" can provide a solution to the incoherence problem by transferring magnetic energy to increasingly larger scales [9]. This mechanism, however, requires a considerable amount of net helicity in the cosmic fluid and therefore scenarios based on inverse cascade are still treated as rather speculative. Inflation has long been suggested as a solution to the causality problem, since it naturally achieves correlations on superhorizon scales. Nevertheless, the conformal invariance of electromagnetism implies that any magnetic field present during the inflationary regime will be strongly diluted by the rapid expansion of the universe. One can get around this obstacle by breaking the conformal invariance of the gauge fields involved $[10,11]$. There are more than one ways of doing that, which explains the variety of the proposed mechanisms in the literature. For example, there have been attempts to create magnetic fields by coupling the photon to a scalar field either during inflation or in the subsequent era of preheating [12]. These proposals have since been criticised in [13]. Other authors have advocated the breakdown of Lorentz invariance either in the context of string theory and non-commutative varying speed of light theories, or due to the dynamics of large extra dimensions [14]. The success of these proposals, however, is usually achieved at the expense of simplicity.

An inflation based mechanism that produces large scale magnetic fields with strengths that could support the dynamo amplification was recently proposed in [4]. One of the attractive aspects of the approach, which exploits the natural coupling between the Z-boson and the gravitational background during inflation, is that it operates within the standard model. The magnitude of the generated magnetic field, however, corresponds to $\sim 10^{-30} \mathrm{G}$ on a collapsed scale of approximately $100 \mathrm{pc}$ today, which is the minimum magnetic strength required for the nonlinear galactic dynamo to operate (in a dark-energy dominated universe). Even when taking into account additional amplification (of up to 5 orders of magnitude) during reheating, the achieved magnetic strength remains rather uncomfortably close to the dynamo margin. Nevertheless, the mechanism proposed in [4] is very promising, as it clearly shows that magnetic fields which survive inflation are not necessarily as weak as previously anticipated.

A common feature in all inflationary models is the production of gravitational radiation with wavelengths extending from about $1 \mathrm{~km}$ to $\sim 3000 \mathrm{Mpc}$ today. In fact, a relic gravity wave spectrum is perhaps the only direct signature of inflation that may still be observable today. The coupling of these inflation produced gravity waves with large scale magnetic fields, which may also be present soon after inflation, could considerably affect the latter. In the present article we try to address this issue within the framework of standard general relativity. We consider the interaction between the aforementioned two sources in a spatially flat FRW cosmology during the radiation and the dust eras. Our results show that, in the presence of gravitational radiation, the magnitude of the magnetic field is amplified proportionally to the shear distortion caused by the propagating waves. Crucially, however, the gravitational boost is also proportional to the square of the field's original scale. This immediately suggests that the mechanism presented here could lead to significant amplification when dealing with large scale magnetic fields. Indeed, when applied to fields of roughly $10^{-34} \mathrm{G}$ spanning a comoving scale of about $10 \mathrm{kpc}$ today, like those produced in [4], our mechanism leads to an amplification of up to 14 orders of magnitude. The 
size of the boost can easily bring these magnetic fields well within the galactic dynamo requirements, without the need for extra amplification during reheating. In fact, the enhancement is so effective that it can bring the field within the dynamo limits even within conventional cosmological models which are not dark-energy dominated. The latter task is more easily achieved when the extra strengthening of the field, due to the adiabatic collapse of the protogalaxy, is also taken into account.

\section{The model}

Consider a spatially flat FRW universe filled with a barotropic perfect fluid and allow for the presence of a weak (test) magnetic field $B_{a}$. For a fundamental observer, moving with 4-velocity $u_{a}\left(u_{a} u^{a}=-1\right)$, the stress-energy tensor of the magnetic field reads $[15,16]$

$$
\mathcal{T}_{a b}=\frac{1}{2} B^{2} u_{a} u_{b}+\frac{1}{6} B^{2} h_{a b}+\Pi_{a b},
$$

where $h_{a b}=g_{a b}+u_{a} u_{b}$ projects orthogonal to $u_{a}$ and $g_{a b}$ is the spacetime metric. Note that in the absence of vorticity $h_{a b}$ is the metric of the hypersurfaces orthogonal to $u^{a}$. Also, $\mathrm{D}_{a}=h_{a}{ }^{b} \nabla_{b}$ defines the covariant derivative operator orthogonal to $u_{a}$. The scalar $B^{2}=B_{a} B^{a}$ measures the energy density and the isotropic pressure of the field, while $\Pi_{a b}=-B_{\langle a} B_{b\rangle}$ describes the anisotropic magnetic pressure. ${ }^{1}$ The weakness of the field implies that its energy density, its anisotropic stresses and its spatial gradients have negligible contribution to the background dynamics. In other words, $B^{2} \ll \rho$ and $\Pi_{a b} \simeq 0 \simeq \mathrm{D}_{b} B_{a}$ to zero order. In this limit, the key background equations are

$$
\begin{aligned}
\kappa \rho-\frac{1}{3} \Theta^{2} & =0, \\
\dot{\rho}+(1+w) \Theta \rho & =0, \\
\dot{B}_{a}+\frac{2}{3} \Theta B_{a} & =0,
\end{aligned}
$$

where $\rho$ is the fluid energy density, $\Theta=3 \dot{a} / a=3 H$ is the expansion scalar (with $a$ and $H$ representing the scale factor and the Hubble parameter respectively) and $w=p / \rho$ (where $p$ is isotropic pressure of the fluid). Equations (2), (3) and (4) are respectively the Friedman equation, the equation of continuity and the magnetic induction equation. For a fully covariant description of electromagnetic fields in an expanding universe the reader is referred to [16]. Finally, we should point out that throughout the paper we employ natural units with $c=1=\hbar$ and $\kappa=8 \pi G=m_{P l}^{-2}$.

We perturb the aforementioned FRW background by allowing for weak gravitational waves, and employ the covariant and gauge-invariant perturbation formalism (see [15]-[19] for details), which guarantees that our results are free from gauge ambiguities. Covariantly, gravity waves are monitored via the electric $\left(E_{a b}\right)$ and the magnetic $\left(H_{a b}\right)$ Weyl components, which are the symmetric and trace-free tensors that describe the long-range gravitational field [17]. In the magnetic presence, one isolates the linear tensor perturbations, namely switches all the scalar and the vector modes off, by imposing the following conditions [18]

$$
\mathrm{D}_{a} B^{2}=0=\varepsilon_{a b c} B^{b} \operatorname{curl} B^{c},
$$

in addition to the standard constraints

$$
\omega_{a}=0=\mathrm{D}_{a} \rho=\mathrm{D}_{a} p,
$$

\footnotetext{
${ }^{1}$ Angled brackets indicate the projected, symmetric and trace-free part of spacelike tensors, while square brackets indicate their antisymmetric part. Furthermore, we will also use $\operatorname{curl} B_{a}=\varepsilon_{a b c} \mathrm{D}^{b} B^{c}$, where $\varepsilon_{a b c}$ is the projected permutation tensor (see [15]).
} 
associated with the pure perfect fluid cosmologies [17]. Together, constraints (5) and (6) guarantee that all traceless tensors are transverse to first order and therefore isolate the pure tensor (i.e. the transverse traceless) modes [18]. Note that condition (5) means that we are dealing with a force-free magnetic field. This in turn implies that any spatial currents that might have been present are also switched off.

\section{The interaction}

Having set the mathematical framework, we now proceed to look into the linear interaction between magnetic fields and propagating gravitational radiation. To first order, the magnetic field evolution in the presence of gravity wave perturbations is governed by the system ${ }^{2}$

$$
\begin{aligned}
\ddot{B}_{a}-\mathrm{D}^{2} B_{a}+\frac{5}{3} \Theta \dot{B}_{a}+\frac{1}{3} \Theta^{2}(1-w) B_{a} & =\frac{4}{3} \Theta \tilde{B}^{b} \sigma_{a b}+2 \tilde{B}^{b} \dot{\sigma}_{a b}, \\
\ddot{\sigma}_{a b}-\mathrm{D}^{2} \sigma_{a b}+\frac{5}{3} \Theta \dot{\sigma}_{a b}+\frac{1}{6} \Theta^{2}(1-3 w) \sigma_{a b} & =0 .
\end{aligned}
$$

Here, $\tilde{B}_{a}$ is the original background magnetic field, and $B_{a}$ is the perturbed field that results from the coupling of $\tilde{B}$ with gravity wave distortions. Thus, to first order, only the background magnetic field contributes to the right-hand side of (7). Note that we have ignored the magnetic contribution to the right-hand side of Eq. (8), given that the linear evolution of the shear is effectively immune to magnetic effects. Indeed, the magnetic presence induces decaying shear modes, which lie in between the standard ones [18]. Also, in deriving Eq. (8) we have employed the linear propagation equation of the shear

$$
\dot{\sigma}_{a b}=-\frac{2}{3} \Theta \sigma_{a b}+\frac{1}{2} \kappa \Pi_{a b}-E_{a b}
$$

which allowed us to express the electric Weyl tensor in terms of $\sigma_{a b}$ and $\Pi_{a b}$. Finally, on using the linear relation $H_{a b}=\operatorname{curl} \sigma_{a b}$, we have replaced the magnetic Weyl tensor with the shear. We should point out that the gravity waves are the driving force behind the "gravito-magnetic" fluctuations in Eq. (7). In particular, one can explicitly show that gravitational waves trigger fluctuations in an otherwise homogeneous background magnetic field distribution via the magnetic part of the Weyl tensor (i.e. via the shear to first approximation) [16].

According to Eq. (4), the background magnetic field evolves as $\tilde{B}_{a}=\tilde{B}_{a}^{0}\left(a_{0} / a\right)^{2}$, where $\dot{\tilde{B}}_{a}^{0}=0$ and the zero suffix corresponds to a given initial time. Assuming that $\tilde{B}_{a}^{0}=\tilde{B}_{(\mathrm{n})}^{0} A_{a}^{(\mathrm{n})}$, with $\dot{A}_{a}^{(\mathrm{n})}=0$ and $\mathrm{D}^{2} A_{a}^{(\mathrm{n})}=-\left(\mathrm{n}^{2} / a^{2}\right) A_{a}^{(\mathrm{n})}$, enables us to define a physical coherence scale $\lambda_{\tilde{B}}=2 \pi a / \mathrm{n}$ for $\tilde{B}_{a}[19]$. Then, introducing the tensor harmonics $Q_{a b}^{(\mathrm{k})}$, with $\dot{Q}_{a b}^{(\mathrm{k})}=0$ and $\mathrm{D}^{2} Q_{a b}^{(\mathrm{k})}=-\left(\mathrm{k}^{2} / a^{2}\right) Q_{a b}^{(\mathrm{k})}$, we adopt the decomposition $\sigma_{a b}=\sigma_{(\mathrm{k})} Q_{a b}^{(\mathrm{k})}$ for the shear. Note that both $A_{a}^{(\mathrm{n})}$ and $Q_{a b}^{(\mathrm{k})}$ are dimensionless and unitary. On these grounds, Eqs. (7) and (8) decompose into the following system of ordinary differential equations:

$$
\begin{aligned}
& \ddot{B}_{(\ell)}+\frac{5}{3} \Theta \dot{B}_{(\ell)}+\left[\frac{1}{3}(1-w) \Theta^{2}+\frac{\ell^{2}}{a^{2}}\right] B_{(\ell)}=2\left(\dot{\sigma}_{(\mathrm{k})}+\frac{2}{3} \Theta \sigma_{(\mathrm{k})}\right) \tilde{B}_{0}^{(\mathrm{n})}\left(\frac{a_{0}}{a}\right)^{2}, \\
& \ddot{\sigma}_{(\mathrm{k})}+\frac{5}{3} \Theta \dot{\sigma}_{(\mathrm{k})}+\left[\frac{1}{6}(1-3 w) \Theta^{2}+\frac{\mathrm{k}^{2}}{a^{2}}\right] \sigma_{(\mathrm{k})}=0,
\end{aligned}
$$

with $B_{a}=B_{(\ell)} B_{a}^{(\ell)}$. In deriving Eq. (10) we have used the vector harmonics $B_{a}^{(\ell)}$, defined by

$$
B_{a}^{(\ell)}=Q_{a b}^{(\mathrm{k})} A_{(\mathrm{n})}^{b},
$$

\footnotetext{
${ }^{2}$ During linearisation the perturbative order of the various quantities is determined by their background value. Quantities with a non-zero unperturbed value have zero perturbative order, while those that vanish in the background are of order one.
} 
which are also dimensionless with an order of unit magnitude. In the above, $\ell$ and $n$ are the comoving wavenumbers of the perturbed and the background magnetic fields, respectively, while $\mathrm{k}$ is the comoving wavenumber of the gravitational wave.

It is rather straightforward to show that $B_{a}^{(\ell)}$ satisfies the standard vector-harmonic requirements. Indeed, starting from definition (12) we find that $\dot{B}_{a}^{(\ell)}=0$, and then $\mathrm{D}^{2} B_{a}^{(\ell)}=-\left(\ell^{2} / a^{2}\right) B_{a}^{(\ell)}$, where $\ell^{2}=\left(\mathrm{k}_{a}+\mathrm{n}_{a}\right)\left(\mathrm{k}^{a}+\mathrm{n}^{a}\right)$. Clearly, the wavenumber $\ell$ provides a measure of the scale of the "induced" magnetic field, which depends on the scale of the background field and on the wavelength of the inducing gravitational radiation. For $\mathrm{n}^{a} \mathrm{k}_{a}=0$, we arrive at the simple expression

$$
\lambda_{B}=\lambda_{\tilde{B}}\left[1+\left(\lambda_{\tilde{B}} / \lambda_{G W}\right)^{2}\right]^{-1 / 2},
$$

for the scale of the perturbed magnetic field. Note that $\lambda_{\tilde{B}}=2 \pi a / \mathrm{n}, \lambda_{G W}=2 \pi a / \mathrm{k}$ and $\lambda_{B}=2 \pi a / \ell$ are the physical wavelengths of the background magnetic field, the gravitational waves and the perturbed magnetic field respectively. According to the above, $\lambda_{B} \leq \lambda_{\tilde{B}}$. In particular, $\lambda_{B} \sim \lambda_{\tilde{B}}$ as long as $\lambda_{G W} \geq \lambda_{\tilde{B}}$, and $\lambda_{B}<\lambda_{\tilde{B}}$ for $\lambda_{G W}<\lambda_{\tilde{B}}$. Here, we will concentrate on large scale magnetic fields and consider their interaction with gravitational radiation of similar or larger wavelength.

Our final step is to introduce the expansion normalized dimensionless variables $\mathscr{B}_{(\ell)}=\kappa^{1 / 2} B_{(\ell)} / \Theta$ and $\Sigma_{(\mathrm{k})}=\sigma_{(\mathrm{k})} / \Theta$, and rewrite Eqs. (10), (11) with respect to conformal time $\eta$ (defined by $\dot{\eta}=a^{-1}$ ) as follows

$$
\begin{gathered}
\mathscr{B}_{(\ell)}^{\prime \prime}+(1-3 w)\left(\frac{a^{\prime}}{a}\right) \mathscr{B}_{(\ell)}^{\prime}-\left[\frac{3}{2}(1-3 w) w\left(\frac{a^{\prime}}{a}\right)^{2}-\ell^{2}\right] \mathscr{B}_{(\ell)}= \\
2 \kappa^{1 / 2} a\left[\Sigma_{(\mathrm{k})}^{\prime}+\frac{1}{2}(1-3 w)\left(\frac{a^{\prime}}{a}\right) \Sigma_{(\mathrm{k})}\right] \tilde{B}_{0}^{(n)}\left(\frac{a_{0}}{a}\right)^{2}, \\
\Sigma_{(\mathrm{k})}^{\prime \prime}+(1-3 w)\left(\frac{a^{\prime}}{a}\right) \Sigma_{(\mathrm{k})}^{\prime}-\left[\frac{3}{2}[1+(2-3 w) w]\left(\frac{a^{\prime}}{a}\right)^{2}-\mathrm{k}^{2}\right] \Sigma_{(\mathrm{k})}=0 .
\end{gathered}
$$

where the prime indicates differentiation with respect to $\eta$.

\section{The effect}

Let us consider the effects described by Eqs. (14) and (15) during different epochs in the lifetime of the universe. For $p=0$, which is the equation of state during dust domination and also the effective equation of state at reheating, $w=0$ and $a^{\prime} / a=2 / \eta$. Then, Eqs. (14) and (15) reduce to

$$
\begin{aligned}
\mathscr{B}_{(\ell)}^{\prime \prime}+\frac{2}{\eta} \mathscr{B}_{(\ell)}^{\prime}+\ell^{2} \mathscr{B}_{(\ell)} & =\frac{8 \alpha_{1}}{\eta^{2}}\left(\Sigma_{(\mathrm{k})}^{\prime}+\frac{1}{\eta} \Sigma_{(\mathrm{k})}\right), \\
\Sigma_{(\mathrm{k})}^{\prime \prime}+\frac{2}{\eta} \Sigma_{(\mathrm{k})}^{\prime}-\left(\frac{6}{\eta^{2}}-\mathrm{k}^{2}\right) \Sigma_{(\mathrm{k})} & =0
\end{aligned}
$$

where $\alpha_{1}=\kappa^{1 / 2} \tilde{B}_{0}^{(\mathrm{n})} / a_{0} H_{0}^{2}$ depends entirely on the initial conditions. In the $\mathrm{k} \eta \ll 1$ limit, namely for long wavelength gravity waves, the solution of Eq. (17) has a dominant mode given by

$$
\Sigma^{(\mathrm{k})}=\Sigma^{(\mathrm{k})}(\eta)=\Sigma_{0}^{(\mathrm{k})}\left(\frac{\eta}{\eta_{0}}\right)^{2}
$$


with $\eta_{0}^{2}=4 / a_{0}^{2} H_{0}^{2}$ (see also [17]). Substituting this result into Eq. (16) we arrive at

$$
\mathscr{B}^{(\mathrm{n})}=\mathscr{B}^{(\mathrm{n})}(\eta)=\mathscr{B}_{0}^{(\mathrm{n})}\left(\frac{\eta_{0}}{\eta}\right)\left[\frac{\cos (\mathrm{n} \eta)}{\cos \left(\mathrm{n} \eta_{0}\right)}\right]+\frac{6 \beta_{1}}{\eta},
$$

where $\beta_{1}=\kappa^{1 / 2} a_{0} \tilde{B}_{0}^{(\mathrm{n})} \Sigma_{0}^{(\mathrm{k})} / \mathrm{n}^{2}$. The first term in the right-hand side of the above solves the homogeneous equation and the second conveys the gravity wave effects. On large scales $n \eta \ll 1$ and (19) reduces to

$$
\mathscr{B}^{(\mathrm{n})}=\mathscr{B}_{0}^{(\mathrm{n})}\left(\frac{\eta_{0}}{\eta}\right)+\frac{6 \beta_{1}}{\eta} .
$$

Note that for large scale gravity waves, with $\lambda_{G W} \sim \lambda_{\tilde{B}}$, we have $\lambda_{B} \sim \lambda_{\tilde{B}}$ and therefore $\ell \sim \mathrm{n}$ (see Eq. (13)). In other words, the wavelength of the perturbed magnetic field effectively coincides with that of the background field. Given that $\mathscr{B}^{(\mathrm{n})}=\kappa^{1 / 2} B^{(\mathrm{n})} / \Theta$ and $B_{0}^{(\mathrm{n})}=\tilde{B}_{0}^{(\mathrm{n})}$ by definition, the above is recast into the following expression for the magnetic field evolution

$$
B^{(\mathrm{n})}=\tilde{B}_{0}^{(\mathrm{n})}\left[1+9\left(\frac{\lambda_{\tilde{B}}}{\lambda_{H}}\right)_{0}^{2} \Sigma_{0}\right]\left(\frac{a_{0}}{a}\right)^{2} .
$$

Here $\left(\lambda_{\tilde{B}}\right)_{0}=a_{0} / \mathrm{n}$ and $\left(\lambda_{H}\right)_{0}=1 / H_{0}$ are the scale of the background magnetic field and the horizon size at a given initial time, respectively. According to (21), the interaction of the background magnetic field with gravity wave distortions can lead to substantial increase of the field if $9\left(\lambda_{\tilde{B}} / \lambda_{H}\right)_{0}^{2} \Sigma_{0} \gg 1$.

Similar effects are also observed during the radiation era. When relativistic species dominate the energy density of the universe $w=1 / 3$ and $a^{\prime} / a=1 / \eta$. Then Eqs. (14), (15) become

$$
\begin{aligned}
\mathscr{B}_{(\ell)}^{\prime \prime}+\ell^{2} \mathscr{B}_{(\ell)} & =\frac{2 \alpha_{2}}{\eta} \Sigma_{(\mathrm{k})}^{\prime}, \\
\Sigma_{(\mathrm{k})}^{\prime \prime}-\left(\frac{2}{\eta^{2}}-\mathrm{k}^{2}\right) \Sigma_{(\mathrm{k})} & =0,
\end{aligned}
$$

where $\alpha_{2}=\kappa^{1 / 2} \tilde{B}_{0}^{(\mathrm{n})} / H_{0}$. For $\mathrm{k} \eta \ll 1$ the solution of $(23)$ has a dominant mode given by $\Sigma^{(\mathrm{k})}=\Sigma_{0}^{(\mathrm{k})} \eta^{2} / \eta_{0}^{2}$, which when substituted into Eq. (22) leads to the solution

$$
\mathscr{B}^{(\mathrm{n})}=\mathscr{B}^{(\mathrm{n})}(\eta)=\mathscr{B}_{0}^{(\mathrm{n})}\left[\frac{\cos (\mathrm{n} \eta)}{\cos \left(\mathrm{n} \eta_{0}\right)}\right]+4 \beta_{2},
$$

with $\beta_{2}=\kappa^{1 / 2} a_{0}^{2} H_{0} \tilde{B}_{0}^{(\mathrm{n})} \Sigma_{0}^{(\mathrm{k})} / \mathrm{n}^{2}$. Confining, as before, to large scale fields only (i.e. $\mathrm{n} \eta \ll 1$ ) we arrive at the following evolution law for the perturbed magnetic field

$$
B^{(\mathrm{n})}=\tilde{B}_{0}^{(\mathrm{n})}\left[1+12\left(\frac{\lambda_{\tilde{B}}}{\lambda_{H}}\right)_{0}^{2} \Sigma_{0}\right]\left(\frac{a_{0}}{a}\right)^{2} .
$$

Results (21), (25) show that the presence of gravity waves does not alter the radiation like evolution of the magnetic field. Thus, dividing either (21) or (25) by the energy density of the background radiation field we obtain

$$
\frac{B}{\rho_{\gamma}^{1 / 2}} \simeq\left[1+10\left(\frac{\lambda_{\tilde{B}}}{\lambda_{H}}\right)_{0}^{2}\left(\frac{\sigma}{H}\right)_{0}\right]\left(\frac{\tilde{B}}{\rho_{\gamma}^{1 / 2}}\right)_{0},
$$


where all the wavenumber indices have now been suppressed. ${ }^{3}$ Expression (26) provides the spectrum of the "comoving" primordial magnetic field, as it would have appeared today were there no galactic collapse and subsequent dynamo amplification. According to (21), (25) and (26), the interaction of the field with gravitational wave distortions can change its magnitude. Crucially, the modification depends not only on the strength of the propagating gravity waves and of the background magnetic field, but also on the scale of the original field. In fact, the effect on the magnitude of the magnetic field is proportional to the square of the ratio $\left(\lambda_{\tilde{B}} / \lambda_{H}\right)_{0}$. Thus, superhorizon-sized magnetic fields, like the ones considered here, interacting with relatively strong gravity waves of comparable or even larger scale can undergo considerable amplification.

The fact that both the perturbed and the background magnetic field share the same (inverse-square) evolution law has two rather important repercussions. First, it ensures that the perturbed field does not alter the gravity wave evolution by inducing any significant new modes (see Eq. (8) and comments immediately bellow that point). Second, it guarantees the "legitimacy" and continuity of Eq. (7) throughout the gravito-magnetic interaction. In other words, the fact that both $B_{a}$ and $\tilde{B}_{a}$ evolve the same ensures that the system $(7),(8)$ also describes the interaction between the gravity waves and $B_{a}$, once the latter has grown strong enough to take over $\tilde{B}_{a}$.

\section{The application}

Hyperhorizon-sized magnetic fields emerge naturally at the end of the inflationary era, as subhorizon quantum fluctuations in the electromagnetic field are stretched out by the rapid expansion of the universe. An attractive mechanism of large scale magnetogenesis, which operates within the standard model, was recently proposed in [4]. In their scenario, the authors exploit the natural coupling between the Z-boson field and the supercooled inflating gravitational background. At reheating, when the EW-symmetry is restored, the Z-boson leads to a (hyper)magnetic field with superhorizon correlations, which converts into a regular magnetic field after the E-W phase transition. The strength of the resulting field, however, is only marginally within the galactic dynamo requirements. In particular, the mechanism proposed in [4] produces a magnetic field of $10^{-30} \mathrm{G}$, on a collapsed scale of $10 \mathrm{kp}$, when redshifted to the epoch of galaxy formation. Although the aforementioned value could increase by up to five orders of magnitude during reheating, $10^{-30} G$ is the minimum seed required for the dynamo amplification to work, and this only if the universe is dominated by a dark energy component (i.e. by a cosmological constant or quintessence) [7]. In addition, the value of $10^{-30} \mathrm{G}$ given in [4] includes the strengthening of the field, by roughly four orders of magnitude, that occurs during the protogalactic collapse. Next we will outline how gravity wave distortions can amplify primordial seed magnetic fields, like those produced in [4], to strengths that lie comfortably within the galactic dynamo requirements.

\footnotetext{
${ }^{3}$ It is worth noticing that at the very long wavelength limit the effect of the gravito-magnetic interaction described by Eqs. (16), (22) closely resembles the superadiabatic amplification of magnetic fields discussed in [11]. The most direct way of demonstrating this resemblance is by dropping the Laplacian terms in (16) and (22). It is then straightforward to show that the dominant magnetic mode no longer evolves as $a^{-2}$. Instead, the field remains constant throughout the radiation dominated epoch, while it decreases proportionally to $a^{-1}$ during the dust (and the reheating) era. In [11], superadiabatic amplification was achieved by introducing an extra coupling between the electromagnetic field and the extrinsic curvature of a spatially flat FRW universe. This broke the conformal invariance of electromagnetism on one hand, but led to a potentially huge increase in the magnetic flux, as hyper-horizon sized fields decayed proportionally to $a^{-1}$ instead of $a^{-2}$. Here we see that, asymptotically, an analogous effect can be achieved through the natural (i.e. the purely relativistic) coupling between the magnetic field and the Weyl curvature of a perturbed FRW model. As we shall see in Sec. 5, the resulting increase of the field, although not as strong as that achieved in [11], can still lead to a considerable boost in the overall magnetic strength.
} 
A collapsed magnetic field of roughly $10^{-30} G$ with size $\sim 100$ pc, corresponds to a field of $\sim 10^{-34} G$ on a comoving scale of about $10 \mathrm{kpc}$ [4]. The interaction of this field with gravitational wave perturbations soon after inflation will boost its magnitude in accordance with (26). The efficiency of the amplification depends on the coherence scale of the field and on the strength of the gravitational waves. Following [4], we apply our analysis to a background magnetic field with comoving strength $\tilde{B} \sim 10^{-34} G$ coherent on a scale of $\sim 10 \mathrm{kpc}$ today. The field's strength corresponds to an energy density ratio $\tilde{B} / \rho_{\gamma}^{1 / 2} \sim 10^{-29}$, which remains constant for as long as the field is frozen into the cosmic medium and the magnetic flux is conserved. Its scale means that $\lambda_{\tilde{B}} / \lambda_{H} \sim 10^{20}$ at the end of inflation, assuming that $H \sim 10^{13} \mathrm{GeV}[4]$. At that time the universe is also permeated by large scale gravitational waves; the inevitable prediction of all inflationary scenarios. In fact, the gravitational wave spectrum generated during the inflationary expansion is perhaps the only direct signature of inflation that might still be observable today. The energy density of these inflation produced gravity waves is given by

$$
\kappa \rho_{G W} \simeq k^{2}\left(\frac{H}{m_{P l}}\right)^{2}
$$

where $k$ is the wave's proper wavenumber and $H$ is the Hubble parameter during inflation (e.g. see [20]). The above translates into the following relation for the wave induced shear anisotropy

$$
\Sigma_{0} \simeq\left(\frac{\lambda_{H}}{\lambda_{G W}}\right)_{0}\left(\frac{H}{m_{P l}}\right)
$$

where the zero suffix indicates the end of the inflationary era. Note that the ratio $H / m_{P l}$ determines the vacuum energy density of the inflaton field and, typically, the lower $H / m_{P l}$ drops the further away from the Planck scale inflation moves. Currently, the quadrupole anisotropy of the CMB constraints $H / m_{P l}$ to be less than $\sim 10^{-5}$, with typical inflationary models having $H / m_{P l} \sim 10^{-6}$. Then, on using expression (28), Eq. (26) becomes

$$
\frac{B}{\rho_{\gamma}^{1 / 2}} \simeq\left[1+10\left(\frac{\lambda_{\tilde{B}}}{\lambda_{H}}\right)_{0}\left(\frac{\lambda_{\tilde{B}}}{\lambda_{G W}}\right)_{0}\left(\frac{H}{m_{P l}}\right)\right]\left(\frac{\tilde{B}}{\rho_{\gamma}^{1 / 2}}\right)_{0} .
$$

Taking $\lambda_{G W} \sim \lambda_{\tilde{B}}$ initially, and substituting the values $\left(\lambda_{\tilde{B}} / \lambda_{H}\right)_{0} \sim 10^{20}$ and $H / m_{P l} \sim 10^{-6}$ into the above, we find that gravity wave perturbations amplify the original field by as much as 13 or 14 orders of magnitude. ${ }^{4}$ This means that $B / \rho_{\gamma}^{1 / 2} \sim 10^{-15}$, which brings the inflationary produced seed of [4] up to $\sim 10^{-21} G$, that is comfortably within the range of the galactic dynamo requirements [7]. Note that the magnitude of $\sim 10^{-21} G$ has been achieved without the need of any extra amplification at reheating or during galaxy formation. Moreover, the efficiency of the amplification also allows us to produce magnetic fields strong enough to sustain the dynamo even in universes with zero cosmological constant. In that case the required value for the ratio $B / \rho_{\gamma}^{1 / 2}$ is raised from $\sim 10^{-25}$ to $\sim 10^{-18}$ and the field itself form $\sim 10^{-30}$ to $\sim 10^{-23} G[6]$. Clearly, the gravitational boost discussed here can also satisfy the latter requirement, especially when the field's enhancement during the protogalactic collapse is also taken into account.

\footnotetext{
${ }^{4}$ Following [4], we are looking at wavelengths that are already far beyond the size of the horizon. This is the reason for the apparently "abrupt" amplification of the field. If, instead, we follow the mode as it leaves the horizon and grows progressively larger, we should see a smoother effect. Note that, as we move on towards larger and larger scales, the magnetic boost described by Eq. (29) corresponds ever more closely to an effective superadiabatic amplification of the field analogous to that discussed in [11] (see also footnote in p. 7).
} 


\section{Discussion}

Magnetic fields have been found everywhere in the universe. Stars, galaxies, clusters of galaxies and even high-redshift formations carry fields that are strong and extensive. The origin of cosmic magnetism. however, is still an unresolved problem. The nonlinear dynamo amplification can provide galactic magnetic fields with the desired strengths of a few $\mu G$, but requires the presence of a seed field to start. So, where did these seeds come from? Two are the main approaches to this question. One appeals to local astrophysical processes, while the other advocates a primordial origin for the seed. The attractive aspect of the latter approach is that it can account for all the observed large scale magnetic fields. Nevertheless, early universe magnetogenesis has its own problems to solve. These have to do with the correlation length and with the strength of the original seed field. Inflation can solve the scale problem but, generally, it leads to magnetic fields that are too weak to sustain the dynamo amplification. Breaking the conformal invariance of the gauge fields involved is the theoretical argument proposed as the way around the strength problem.

Recently, an attractive inflation based mechanism, which operates within the standard model, was suggested for the production of large scale primordial magnetic fields [4]. The strength of these fields is within the galactic dynamo requirements, albeit for dark energy dominated universes and only marginally. Even when additional amplification during the brief period of reheating is taken into account, the achieved magnitudes are still relatively close to the dynamo lower limits. Here, we have outlined the basic features of a mechanism which can amplify these inflationary produced magnetic fields to strengths that lie well within the galactic dynamo requirements, even in conventional cosmologies with zero dark energy component. Our approach is based on standard general relativity and considers the interaction of large scale primordial magnetic fields with gravitational wave distortions in the post inflationary era. Gravity waves are inevitable byproducts in all inflationary models, with a spectrum extending over a very wide range of wavelengths. By allowing these waves to interact with magnetic fields of comparable size, we found a considerable amplification in the magnitude of the fields. As expected, the gravitational wave boost was proportional to the induced shear anisotropy. Crucially, however, the amplification is also proportional to the square of the magnetic field's original coherence length. This immediately suggested that there could be strong gravitational amplification of magnetic fields spanning hyperhorizon scales, like those produced in [4]. When the actual numbers were inserted in the equations, we found that the gravity wave induced amplification could reach up to 13 or 14 orders of magnitude. The size of the boost easily brings the primordial fields produced in [4] up to strengths of $10^{-21}-10^{-20} \mathrm{G}$, without the need of any additional amplification at reheating or during galaxy formation. These values are well within the dynamo limits associated with spatially flat dark-energy dominated universes. Moreover, when the effect of protogalactic collapse is also taken into account, the achieved magnetic strengths lie comfortably within the dynamo requirements associated, this time, with conventional (i.e. zero dark-energy) cosmologies.

\section{Acknowledgments}

We would like to thank John Barrow, Andrew Liddle and Roy Maartens for helpful discussions and comments. The authors also acknowledge support from a Sida/NRF grant. 


\section{References}

[1] P.P. Kronberg 1994 Rep. Prog. Phys. 57 325; J-L Han and R. Wielebinski 2002 Chin. J. Astron. Astrophys. 2293

[2] D. Grasso and H. Rubinstein 2001 Phys. Rep. 348 163; L.M. Windrow 2002 Rev. Mod. Pys. 74775

[3] E.R. Harrison 1970 M.N.R.A.S. 147 279; C.J. Hogan 1983 Phys. Rev. Lett. 51 1488; T. Vachaspati 1991 Phys. Lett. B 265 258; M. Gasperini, M. Giovanini and G. Veneziano 1995 Phys. Rev. Lett. 75 3796; G. Sigl, K. Jedamzik and A.V. Olinto 1997 Phys. Rev. D 55, 4582; M. Joyce and M. Shaposhnikov 1997 Phys. Rev. Lett. 79 1193; E. Calzetta and A. Kandus 2002 Phys. Rev. D 65 063004

[4] A-C Davis, K. Dimopoulos, T. Prokopec and O. Törnkvist 2001 Phys. Lett. B 501 165; K. Dimopoulos, T. Prokopec, O. Törnkvist and A-C. Davis 2002 Phys. Rev. D 65063505

[5] E.N. Parker Cosmical Magnetic Fields (Clarendon, Oxford 1979); Y.B. Zeldovich, A.A. Ruzmaikin and D.D. Sokoloff Magnetic Fields in Astrophysics (McGraw-Hill, New York 1983); P.P. Kronberg 1994 Rep. Prog. Phys. 57 325; R. Beck, A. Brandenburg, D. Moss, A.A. Shukurov and D. Sokoloff 1996 Annu. Rev. Astron. Astrophys. 34155

[6] R.M. Kulsrud, in Galactic and Extragalactic Magnetic Fields, Eds R. Beck, P.P. Kronberg, and R. Wielebinski (Reidel, Dordrecht, 1990).

[7] A.C. Davis, M. Lilley and O. Törnkvist 1999 Phys. Rev. D 60021301

[8] R.M. Kulsrud and S.W. Anderson 1992 Astrophys. J. 396606

[9] J.M. Cornwall 1997 Phys. Rev. D 56 6146; D.T. Son 1999 Phys. Rev. D 59 063008; G.B. Field and S.M. Carroll 2000 Phys. Rev. D 62 103008; M. Christensson, M. Hindmarsh and A. Brandenburg 2001 Phys. Rev. E 64056405

[10] A.D. Dolgov 1981 Sov. Phys. JETP 54 223; A.D. Dolgov 1993 Phys. Rev. D 482499

[11] M.S. Turner and L.M. Windrow 1988 Phys. Rev. D 302743

[12] E.A. Calzetta, A. Kandus and F.D. Mazzitelli 1998 Phys. Rev. D 57 7139; A. Kandus, E.A. Calzetta, F.D. Mazzitelli and C.E.M. Wagner 2000 Phys. Lett. B 472 287; B.A. Bassett, G. Pollifrone, S. Tsujikawa and F. Viniegra 2001 Phys. Rev. D 63103515

[13] M. Giovannini and M. Shaposhnikov 2000 Phys. Rev. D 62103512

[14] O. Bertolami and D.F. Mota 1999 Phys. Lett. B 455 96; A. Mazumdar, M.M. Sheikh-Jabbari 2001 Phys. Rev. Lett. 87 011301; M. Giovannini 2000 Phys. Rev. D 62123505

[15] G.F.R. Ellis, in Cargèse Lectures in Physics, vol. 1, edited by E. Schatzman (Gordon and Breach, New York, 1973) p. 1; G. F. R. Ellis and H. van Elst, in Theoretical and Observational Cosmology, edited by M. Lachièze-Rey (Dordrecht: Kluwer 1999) p. 1 
[16] C.G. Tsagas and J.B. Barrow 1997 Class. Quantum Grav. 14 2539; C.G. Tsagas and J.D. Barrow 1998 Class. Quantum Grav. 15 3523; C.G. Tsagas and R. Maartens 2000 Phys. Rev. D 61083519 ; C.G. Tsagas and R. Maartens 2000 Class. Quantum Grav. 172215

[17] P.K.S. Dunsby, B.A.C.C. Bassett and G.F.R. Ellis 1997 Class. Quantum Grav. 14 1215; A. Challinor 2000 Class. Quantum Grav. 17871

[18] R. Maartens, C.G.Tsagas and C. Ungarelli 2001 Phys. Rev. D 63 123507; C.G. Tsagas 2002 Class. Quantum Grav. 193709

[19] M. Marklund, P. K. S. Dunsby and G. Brodin 2000 Phys. Rev. D 62104008

[20] E.W. Kolb and M.S. Turner The Early Universe (Addison-Wesley 1990); T. Padmanabhan Structure Formation in the Universe (Cambridge University Press, Cambridge 1993); J.A. Peacock Cosmological Physics (Cambridge University Press, Cambridge 1999); A.R. Liddle and D.H. Lyth Cosmological Inflation and Large-Scale Structure (Cambridge University Press, Cambridge 2000) 